\title{
Return-current formation in the electron beam - plasma system
}

\author{
M. Karlický and M. Bárta \\ Astronomical Institute, Academy of Sciences of the Czech Republic, 25165 Ondřejov, Czech Republic
}

Received: 4 May 2009 - Revised: 19 July 2009 - Accepted: 20 July 2009 - Published: 23 July 2009

\begin{abstract}
Using a 3-D electromagnetic particle-in-cell model an evolution of the electron distribution function in the beam-plasma system with the return current is computed. It was found that the resulting electron distribution function depends on the magnetic field assumed along the beampropagation direction. While for small magnetic fields the electron distribution function becomes broad in the direction perpendicular to the beam propagation due to the Weibel (filamentation) instability and the return current is formed by a shifted bulk distribution, for stronger magnetic fields the distribution, especially on the return current side, is extended in the beam-propagation direction. To understand better the instabilities influencing the mentioned processes, the dispersion diagrams are computed and discussed.
\end{abstract}

\section{Introduction}

It is commonly believed that electron beams accelerated in solar flares bombard dense layers of the solar atmosphere where they are decelerated and produce hard X-ray emissions (Brown et al., 1990). The electron beams with the estimated fluxes of $F_{E}=10^{9}-10^{12} \mathrm{ergs} \mathrm{s}^{-1} \mathrm{~cm}^{-2}$ carry huge electric currents that have to be neutralized by the return currents (Hoyng et al., 1978; van den Oord, 1990). There are two analytical concepts of the return current: (a) the return current is formed by all background plasma electrons having the same drift velocity - the Maxwell electron distribution is shifted in the velocity space compared to the protons (e.g. Karlický and Henoux 1992), and (b) Rowland and Vlahos (1985) proposed that the return current is formed by socalled runaway electrons. Formation of the return current has two physical aspects: (a) electric charge effects at the front

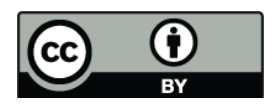

\section{Correspondence to: M. Karlický}

(karlicky@asu.cas.cz) of the electron beam and (b) electromagnetic effects of the beam electric current (van den Oord, 1990).

Return-current effects on the dynamics of nonthermal electron beams and the heating of the flare atmosphere have been described and studied by Emslie (1980), Karlický et al. (1990), Karlický and Henoux (1992), and Zharkova et al. (1995). In the papers by Karlický and Henoux (2002), Henoux and Karlický (2003), Karlický et al. (2004), Štepán et al. (2007) it was proposed that the polarization and enhanced intensities of the chromospheric optical lines can be explained by a formation of the return current at chromospheric layers. It was found that the resulting intensities and polarization are very sensitive to the form of the electron distribution function (with the return current), especially in the range of the excitation and ionization energies of these lines. Furthermore, the return current modifies the electron distribution function in the low corona and influences the intensities of the EUV and soft X-ray lines observed during solar flares (see Dzifčáková and Karlický, 2008).

In all the above mentioned papers the return current have been considered on time scales (seconds) much longer than those for the formation of the return current, and thus various approximations of the return current have been used. But now a much more detailed knowledge of the electron distribution function in the beam-plasma system is demanded, not only in the solar flare research, but everywhere the electron beams are studied.

Therefore in this paper, we study an evolution of the electron beam in the background plasma with the return current using a 3-D particle-in-cell (PIC) electromagnetic code. Especially, we are interested about an influence of the magnetic field assumed along the beam-propagation direction. This paper is a natural extension of our previous papers where an importance of the Weibel instability was recognized (Karlický et al., 2008; Karlický, 2009). Note that the effects of magnetic field on the Weibel instability have been investigated for relativistic jets by Hededal and Nishikawa (2005).

Published by Copernicus Publications on behalf of the European Geosciences Union and the American Geophysical Union. 

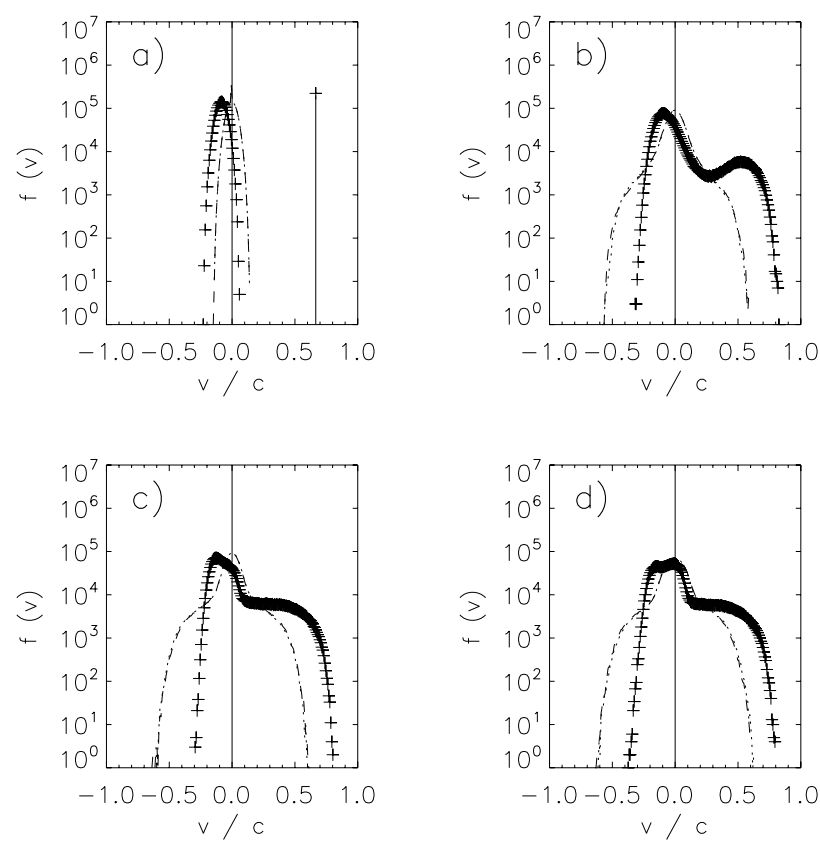

Fig. 1. The electron distribution functions in the whole numerical box (the crosses means $f\left(v_{z}\right)$, dot-dashed line means $f\left(v_{x}\right)$ or $\left.f\left(v_{y}\right)\right)$ at four different times: at the initial state (a), at $\omega_{p e} t=40$ (b), at $\omega_{p e} t=100$ (c), and $\omega_{p e} t=200$ (d). The line in the part a) at $v / c=0.666$ means the monoenergetic electron beam (the $\delta$ function). In the initial state the magnetic field corresponding to the ratio $\omega_{c e} / \omega_{p e}=0.1$ is considered.

Remark: The Weibel instability in the sense as used in these papers is also designated as the filamentation instability (Bret et al., 2005; Bret, 2009).

\section{Model}

For our study we used a 3-D (3 spatial and 3 velocity components) relativistic electromagnetic PIC code (Buneman, 1993). The system size is $L_{x}=45 \Delta, L_{y}=45 \Delta$ and $L_{z}=600 \Delta$ (where $\Delta$ is the grid size).

We initiated a spatially homogeneous electron-proton plasma with the proton-electron mass ratio $m_{p} / m_{e}=16$. (For comparison we made also some computations with $m_{p} / m_{e}=100$.) This is unrealistic and it was chosen to shorten the proton skin depth and computations. Nevertheless, the ratio is still sufficient to separate the dynamics of electrons and protons well. The electron thermal velocity is $v_{T e}=0.06 c$ (the corresponding temperature is $T_{e}=21.4 \mathrm{MK}$ ), where $c$ is the speed of light. In all models, 160 electrons and 160 protons per cube grid were used. The plasma frequency is $\omega_{p e}=0.05$, and the electron Debye length is $\lambda_{D}=0.6 \Delta$. The electron and proton skin depths are $\lambda_{c e}=10 \Delta$ and $\lambda_{c i}=40 \Delta$, respectively.
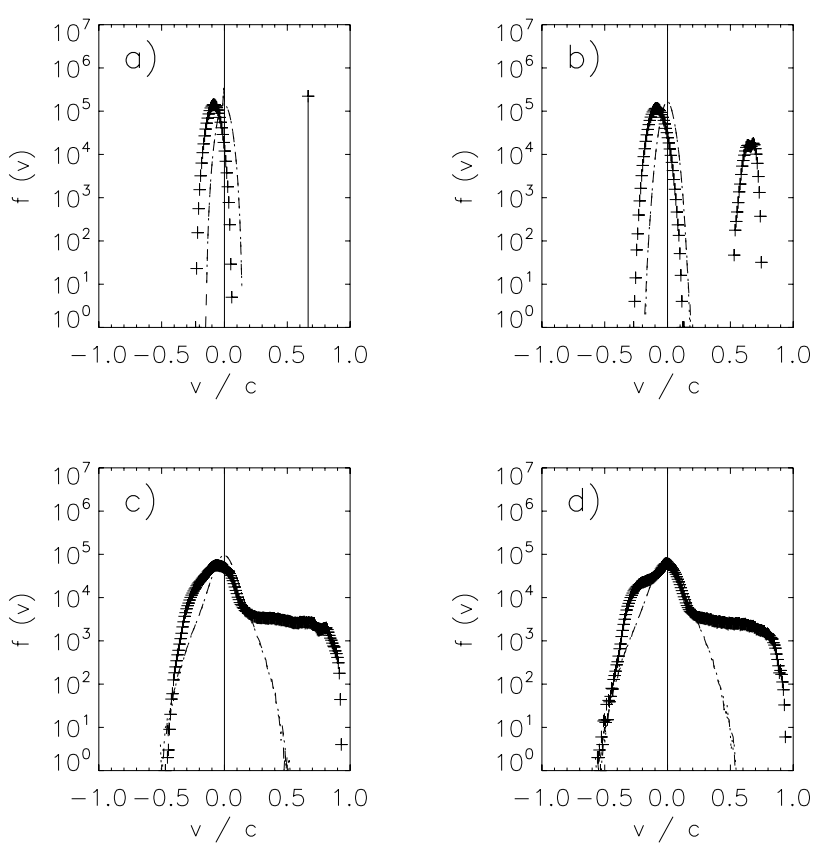

Fig. 2. The electron distribution functions in the whole numerical box (the crosses means $f\left(v_{z}\right)$, dot-dashed line means $f\left(v_{x}\right)$ or $\left.f\left(v_{y}\right)\right)$ at four different times: at the initial state (a), at $\omega_{p e} t=40$ (b), at $\omega_{p e} t=100$ (c), and $\omega_{p e} t=200$ (d). The line in the part a) at $v / c=0.666$ means the monoenergetic electron beam. In the initial state the magnetic field corresponding to the ratio $\omega_{c e} / \omega_{p e}=0.7$ is considered.

Then, we included one monoenergetic beam (homogeneous in the whole numerical box). To keep the total current to be zero in these models in the initial states, we shifted the background plasma electrons in the velocity space according to the relation $v_{D}=-v_{b} n_{b} / n_{e}$, where $v_{b}$ is the velocity of the electron beam, $\mathrm{n}_{b}$ and $\mathrm{n}_{e}$ are the beam and background plasma densities (for this type of initiation, see also Niemiec et al., 2008). The beam velocity was chosen to be $v_{b} / c=2 / 3$ (in the $z$ direction). The ratio of the beam and plasma densities was taken as $n_{b} / n_{e}=1 / 8$.

Because we wanted to know an effect of the magnetic field, we varied the ratio of electron-cyclotron and electronplasma frequencies. The periodic boundary conditions were used.

All computations were performed on the parallel computer OCAS (Ondrejov Cluster for Astrophysical Simulations), see http://wave.asu.cas.cz/ocas.

\section{Results of 3-D PIC simulations}

As an illustration of the time evolution of the electron distribution function in the beam-plasma system with the return current, Fig. 1 shows the evolution of the system with low magnetic field $\left(\omega_{c e} / \omega_{p e}=0.1\right)$ parallel to the beam 

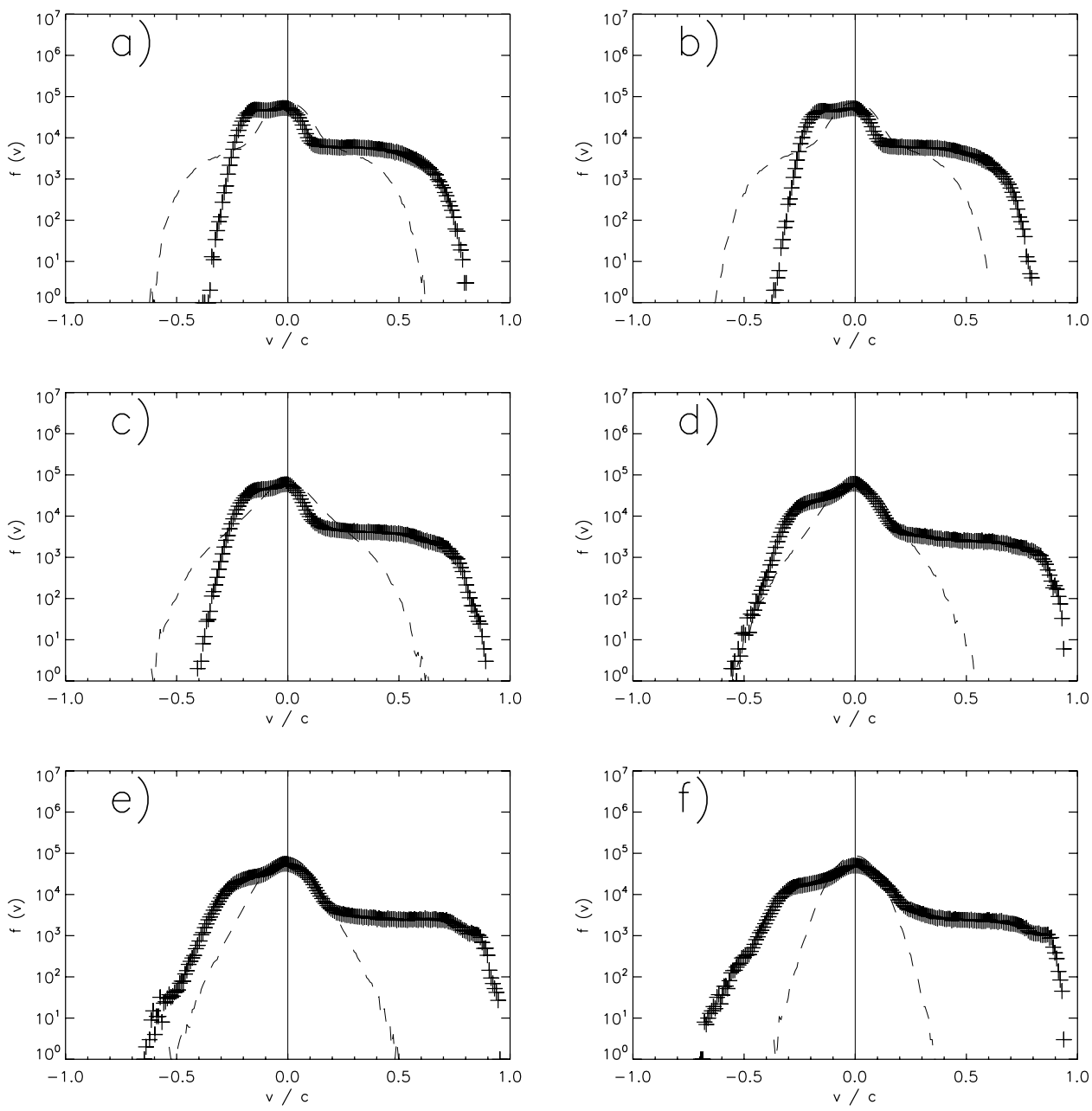

Fig. 3. The electron distribution functions in the whole numerical box (the crosses means $f\left(v_{z}\right)$, dashed line means $f\left(v_{x}\right)$ or $\left.f\left(v_{y}\right)\right)$ at $\omega_{p e} t=200$ in dependence on the magnetic field expressed through the ratio $\omega_{c e} / \omega_{p e}=0.0$ (a), 0.1 (b), 0.5 (c), 0.7 (d), 1.0 (e), and 1.3 (f), respectively.

propagation direction. As can be seen here due to the beamplasma instability (Michailovskij, 1975) the plateau on the distribution function $f\left(v_{z}\right)$ (in the beam propagation direction) on the beam side is formed. Simultaneously, the distribution functions $f\left(v_{x}\right)$ and $f\left(v_{y}\right)$, i.e. the distribution functions in the perpendicular directions to that of the beam propagation, are strongly heated. It is due to the Weibel instability (1959) (see also the paper by Nishikawa at al., 2006). The return current in this case is characterized by a broad and shifted bulk of the distribution function.

In a further example, we increased the magnetic field strength to that corresponding to the ratio of the electroncyclotron and electron-plasma frequency $\omega_{c e} / \omega_{p e}=0.7$. A time evolution of the electron distributions in this case is shown in Fig. 2.

To see in more detail how the magnetic field influences the resulting electron distribution function (at the time $\left.\omega_{p e} t=200\right)$, Fig. 3 presents the distribution functions for six values of the ratio of the electron-cyclotron and electronplasma frequencies $\left(\omega_{c e} / \omega_{p e}=0.0,0.1,0.5,0.7,1.0\right.$, and 1.3). It is evident that with an increase of the ratio $\omega_{c e} / \omega_{p e}$ the role of the Weibel (filamentation) instability is more and more reduced, the distribution functions in the perpendicular direction to that of the beam propagation $f\left(v_{x}\right)$ and $f\left(v_{y}\right)$ are less heated. On the other hand, the problem of the return current formation becomes more and more one-dimensional and more extended tail on the return current side is formed (compare Fig. 3a and f) (see also Karlický et al., 2008 and Karlický, 2009).

Furthermore, in Fig. 4 we present a comparison of the beam-plasma systems with two different beam velocities $\left(v_{b} / c=2 / 3\right.$ and 1/3). As seen here the basic aspects of the evolutions remain the same. 

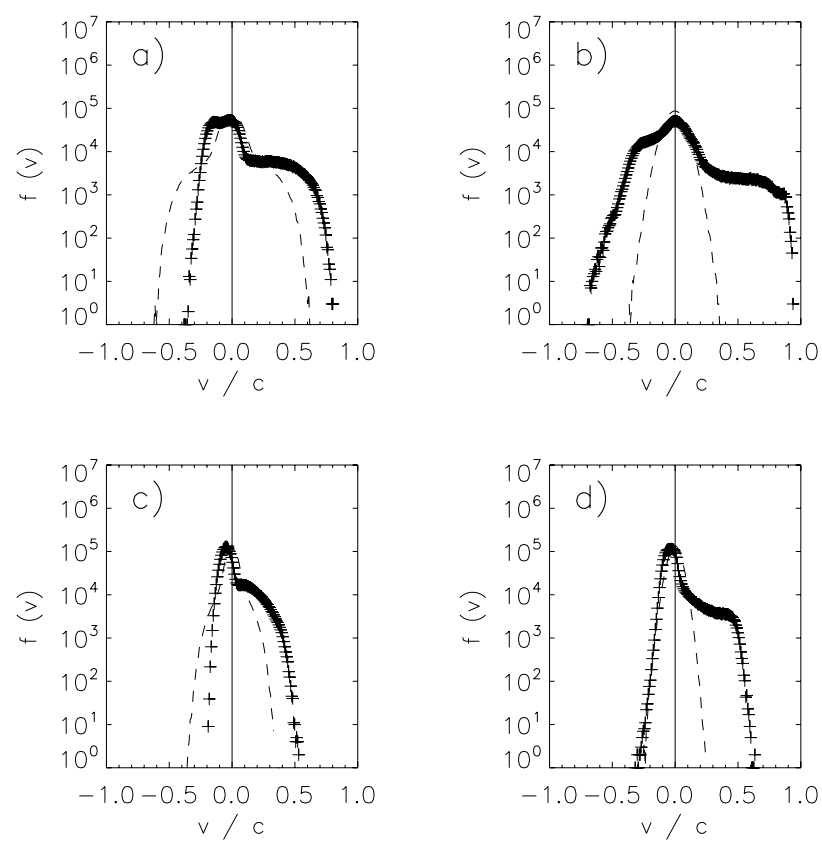

Fig. 4. The electron distribution functions in the whole numerical box (the crosses means $f\left(v_{z}\right)$, dot-dashed line means $f\left(v_{x}\right)$ or $\left.f\left(v_{y}\right)\right)$ at $\omega_{p e} t=200$ for two different beam velocities $\left(v_{b} / c=2 / 3\right.$ (a and $\mathbf{b}), v_{b} / c=1 / 3$ (c and d)) and two different ratio $\omega_{c e} / \omega_{p e}=0$ (a and $\mathrm{c}$ ), $\omega_{c e} / \omega_{p e}=1.3$ (b and d).

Table 1. The estimated growth rates for the two-stream and filamentation instabilities in dependance on the magnetic field.

\begin{tabular}{ccc}
\hline$\omega_{c e} / \omega_{p e}$ & $\gamma_{t w o} / \omega_{p e}$ & $\gamma_{f i l} / \omega_{p e}$ \\
\hline 0.0 & 0.11 & 0.23 \\
0.5 & 0.14 & 0.15 \\
1.3 & 0.21 & 0.08 \\
\hline
\end{tabular}

Now, to understand better what instabilities are dominant in the evolution of the systems under study, we made the diagnostics as follows:

In Figs. 5 and 6 we compare the time evolution of the electric field energy in the whole numerical box in the $\mathrm{z}$ coordinate (the electrostatic wave energy) and the magnetic field energy in the perpendicular direction to that of the beam propagation for three values of the ratio $\omega_{c e} / \omega_{p e}=0,0.5$, and 1.3. As seen here, while the role of the two-stream instability increases with the increase of the magnetic field, the importance of the Weibel (filamentation) decreases. It is due to the fact that the magnetic field stabilizes the beam against perpendicular perturbations. Using results presented in Figs. 5 and 6 we estimated the growth rates of both the instabilities and results are summarized in Table 1. For comparison the growth rate of the cold beam - plasma instability is given by the relation (Michailovskij, 1975):

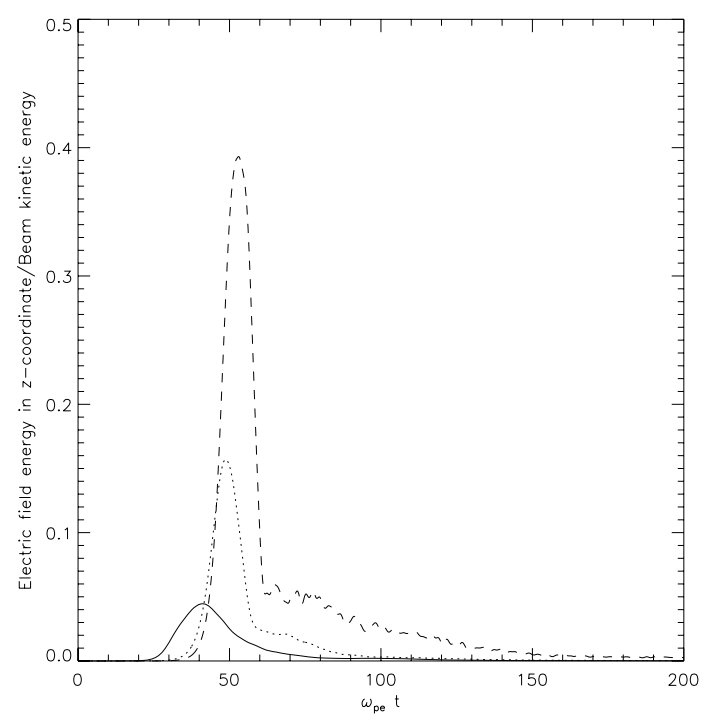

Fig. 5. The electric field energy in the z-coordinate for three values of the ratio $\omega_{c e} / \omega_{p e}=0$ (full line), 0.5 (dotted line), and 1.3 (dashed line).

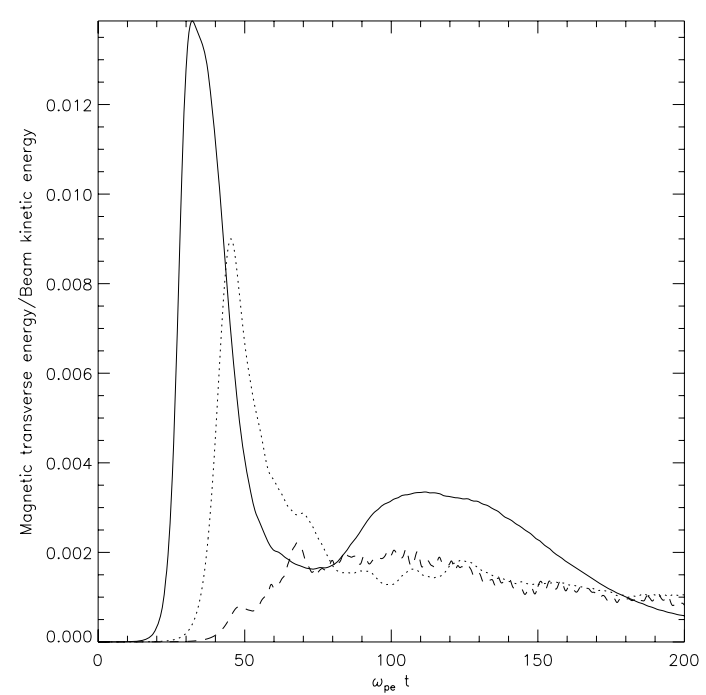

Fig. 6. Magnetic field energy in the perpendicular direction to the beam propagation for three values of the ratio $\omega_{c e} / \omega_{p e}=0$ (full line), 0.5 (dotted line), and 1.3 (dashed line).

$\gamma / \omega_{p e}=\sqrt{3}\left(n_{b} / n_{e}\right)^{1 / 3} / 2^{4 / 3}$

which in our case is $\gamma / \omega_{p e}=0.34$. It means that in all three cases the computed growth rates are smaller than that derived from the analytical relation. Nevertheless, the best agreement is in the case of the strongest magnetic field $\left(\omega_{c e} / \omega_{p e}=1.3\right)$. On the other hand, the growth rates of the filamentation instability decreases with the magnetic field increase. 

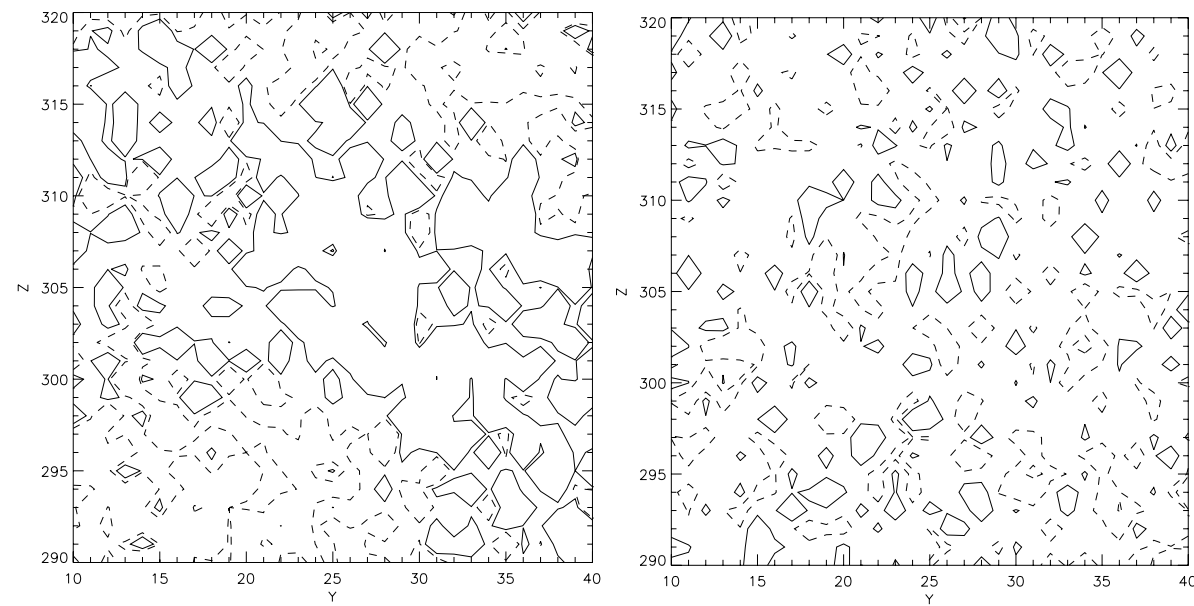

Fig. 7. Examples of the structure of the electric current ( $j_{x}$ component) (the full and dashed contours mean positive or negative values) at $\omega_{p e} t=40$ for two values of the ratio $\omega_{c e} / \omega_{p e}=0$ (left panel) and 1.3 (right panel). The $\mathrm{y}$ - and $\mathrm{z}$-coordinates are in grids.
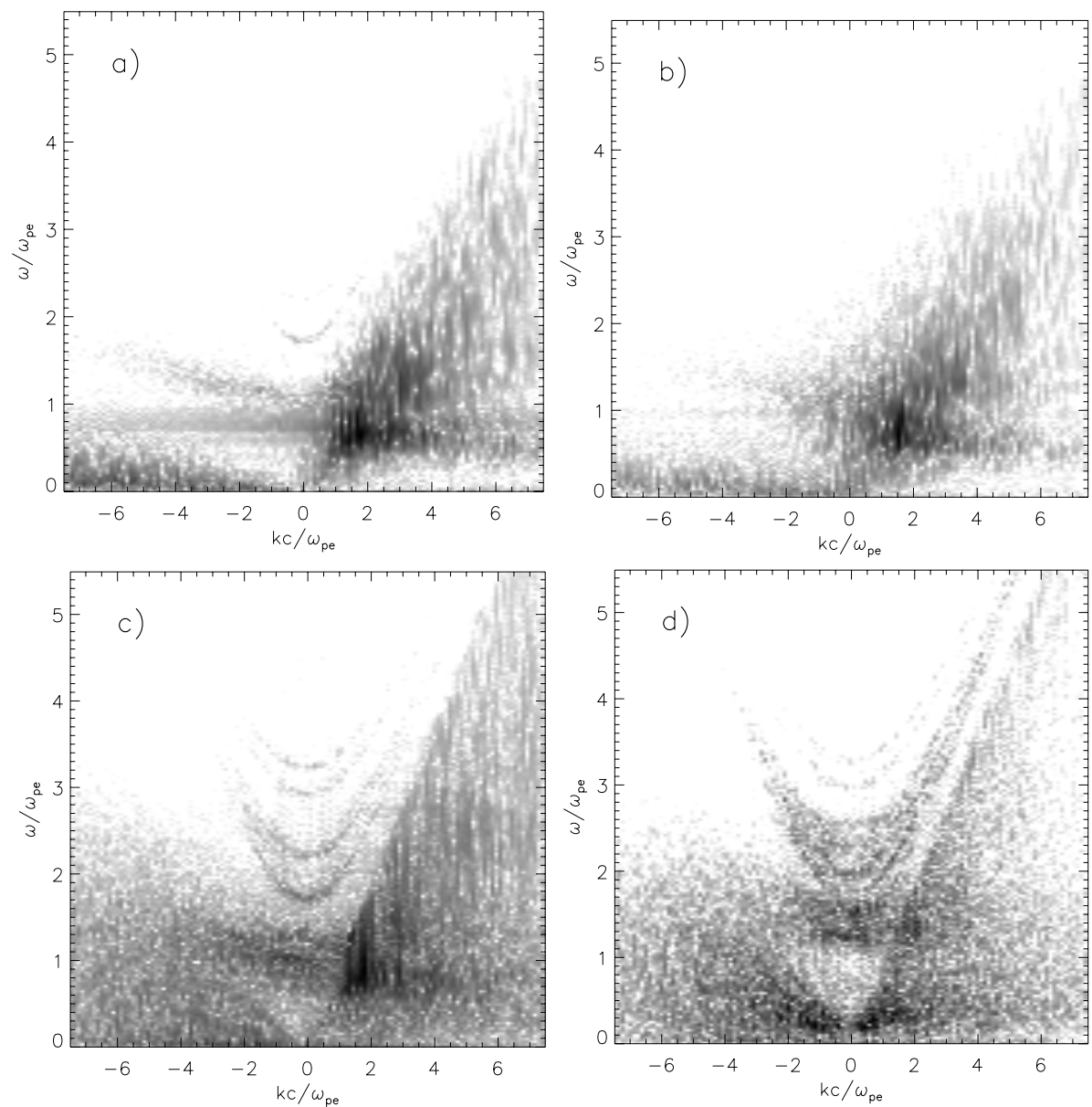

Fig. 8. The dispersion diagrams for the electric field $E_{z}$ (a and $\mathbf{c}$ ) and $E_{x}$ (or $E_{y}$ ) (b and $\mathbf{d}$ ) for $k$ parallel to the magnetic field and electron beam propagation (z-coordinate) in the time interval $\omega_{p e} t=0-200$ for two values of the ratio $\omega_{c e} / \omega_{p e}=0.0(\mathbf{a}$ and $\mathbf{b})$ and 1.3 (c and $\left.\mathbf{d}\right)$. The mass ratio $m_{i} / m_{e}=16$. The black color means energetic wave modes. 

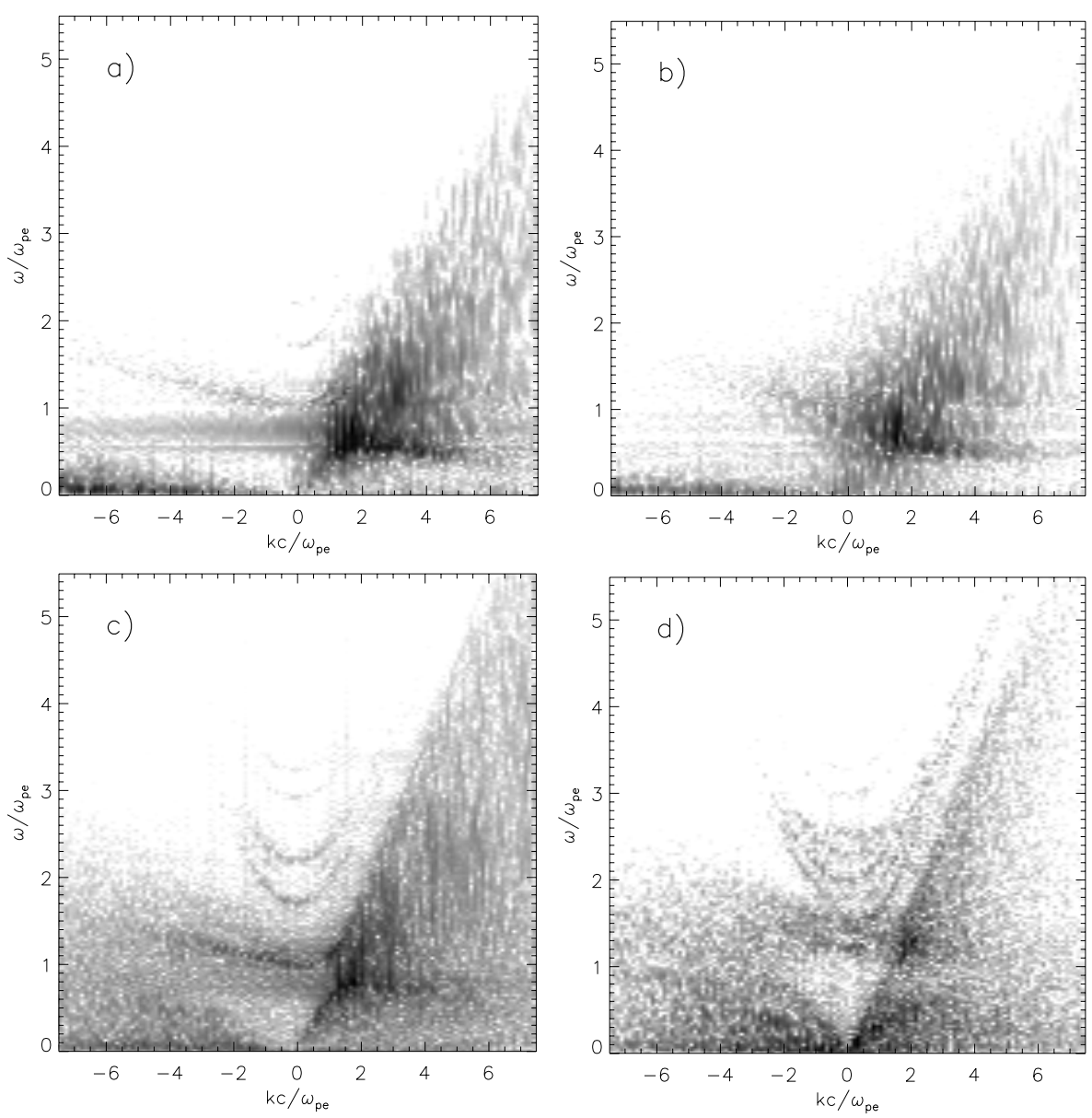

Fig. 9. The dispersion diagrams for the electric field $E_{z}$ (a and $\mathbf{c}$ ) and $E_{x}$ (or $E_{y}$ ) (b and d) for $k$ parallel to the magnetic field and electron beam propagation (z-coordinate) in the time interval $\omega_{p e} t=0-200$ for two values of the ratio $\omega_{c e} / \omega_{p e}=0.0$ (a and $\left.\mathbf{b}\right)$ and 1.3 (c and $\left.\mathbf{d}\right)$. The mass ratio $m_{i} / m_{e}=100$.

In all cases presented in Figs. 5 and 6, first, the energies exponentially increase (linear phase of instabilities) and then after their saturation they decrease (nonlinear phases). In Fig. 6 it is interesting to see that the magnetic field energy has a second maximum in the nonlinear phase of the Weibel (filamentation) instability.

Generally the saturation maxima of the magnetic field energies (Fig. 6) are much lower than those of the electric field energies (Fig. 5). Nevertheless, it is interesting that for low values of $\omega_{c e} / \omega_{p e}=0$ and 0.5 the magnetic field energies grow earlier than the electric field energies. Both the mentioned instabilities compete and interact in the studied systems. Another evidence of the presence of the Weibel (filamentation) instability is the formation of structured electric currents, see Fig. 7, where an example of such a structure in the model with zero magnetic field (left panel) is compared with the case with no such a structure (right panel) (see similar structures in the paper by Nishikawa et al., 2003).
Because in our systems the velocity of the shifted Maxwell distribution of background plasma electrons $v_{D}$ is greater than the thermal velocity, the return current can be unstable even for the Buneman instability. Furthermore, there exist further instabilities which can play a role in the studied system, see the extended analysis by Bret (2009). In later phases of evolution of the system even electrostatic double layers can be generated and thus influencing a transport of energetic electrons through the plasma (Lee et al., 2007, 2008).

The dispersion diagrams shown in Figs. 8 (the case with $m_{i} / m_{e}=16$ ) and 9 (the case with $m_{i} / m_{e}=100$ ) confirm the complexity of processes in the studied systems. These diagrams were computed from the electric field components recorded along the beam propagation direction, along the line with $x=22 \Delta$ and $y=22 \Delta$, i.e. the k-vector in Figs. 8 and 9 is oriented along the $\mathrm{z}$-coordinate. The branches of generated waves (expressed by the black color) look to be more distinct for the case with $m_{i} / m_{e}=100$. It is probably due to the fact that the proton and electron modes for higher mass ratio are 
more separated, or the change of $m_{i} / m_{e}$ causes that the maximum growing modes are better fitted with chosen numerical grids. The strongest wave modes generated are the Langmuir waves, see the black feature close to $\omega / \omega_{p e} \sim 0.8$ and $k c / \omega_{p e} \sim 1.5$ (a crossing of the Langmuir and beam branches) in agreement with the resonance condition: $\omega_{p e} \approx k v_{b}$, where $v_{b}$ is the beam velocity (see Figs. $8 \mathrm{a}$ and $9 \mathrm{a}$ ). In the cases with the magnetic field the electromagnetic branches appeared (see Figs. 8c, d, 9c, and d at the frequencies greater $\left.\omega_{p e}\right)$. Some wave energy is accumulated also in low frequency waves $\left(\omega / \omega_{p e} \leq 1\right)$ (Figs. $8 \mathrm{~d}$ and $\left.9 \mathrm{~d}\right)$. Even it looks that the whistler waves are generated, see branches in the region $\omega / \omega_{p e} \leq 1$ and $k c / \omega_{p e} \sim(-3-3)$ in Fig. $9 \mathrm{~d}$.

\section{Conclusions}

The presented results show that the beam-plasma system with the return current is under play of many instabilities: two-stream, Weibel (filamentation), current-driven and so on in agreement with the analysis made by Bret (2009) and Lee et al. $(2007,2008)$. Due to these instabilities the electron distribution function and return current evolve. While the two-stream instability is important in all presented cases, the filamentation instability is reduced for the strong magnetic field $\left(\omega_{c e} / \omega_{p e} \approx 1\right)$. On the other hand, for weak magnetic fields $\left(\omega_{c e} / \omega_{p e} \leq 0.1\right)$ the filamentation instability strongly heats the electrons in the direction perpendicular to that of the beam propagation. Simultaneously, the distribution function of the return current changes from that with the extended tail (for strong magnetic fields) to the return current formed by a broad and shifted bulk of the electron distribution function (for weak magnetic fields). Furthermore it was found that for the cases with strong magnetic field also low frequency modes are generated (whistlers?). But a disentangling of these modes and understanding of their roles in the studied processes need more sophisticated models.

Finally, we can conclude that the found variations of the distribution functions should influence the intensities of EUV and soft X-ray lines formed in the fully ionized plasma in the coronal parts of solar flares.

Acknowledgements. This research was supported by the Center for Theoretical Astrophysics, Prague and by Grant IAA300030701 of the Grant Agency of the Academy of Sciences of the Czech Republic. The author thanks to all three referees for constructive comments that improved the paper.

Edited by: J. Büchner

Reviewed by: K.-I. Nishikawa and another anonymous referee

\section{References}

Bret, A.: Weibel, two-stream, filamentation, oblique, Bell, Buneman, Which one grows faster?, Astrophys. J., 699, 990-1003, 2009.

Bret, A., Firpo, M.-C., and Deutsch, C.: Characterization of the initial filamentation of a relativistic electron beam passing through a plasma, Phys. Rev. Lett., 94, 115002-115005, 2005.

Brown, J. C., Karlický, M., MacKinnon, A. L., and van den Oord, G. H. J.: Beam heating in solar flares - electrons or protons?, Astrophys. J. Suppl. Ser., 73, 343-348, 1990.

Buneman, O.: TRISTAN, in Computer Space Plasma Physics, edited by: Matsumoto, H. and Omura, Y., Terra Scientific Publ Comp., Tokyo, 305-322, 1993.

Dzifčáková, E. and Karlický, M.: Influence of the return current on the EUV and X-ray flare line emissions, Sol. Phys., 250, 329338, 2008.

Emslie, A. G.: The effect of reverse currents on the dynamics of nonthermal electron beams in solar flares and on their emitted X-ray bremsstrahlung, Astrophys. J., 235, 1055-1065, 1980.

Hededal, C. B. and Nishikawa, K.-I.: The influence of an ambient magnetic field on relativistic collisionless plasma shocks, Astrophys. J., 623, L89-L92, 2005.

Hénoux, J. C. and Karlický, M.: First detection of return currents in solar flares by spectropolarimetry with THEMIS, Astron. Astrophys., 407, 1103-1113, 2003.

Hoyng, P., Knight, J. W., and Spicer, D. S.: Diagnostics of solar flare hard X-ray sources, Sol. Phys., 58, 139-148, 1978.

Karlický, M.: Electron beam-plasma interaction and the returncurrent formation, Astrophys. J., 690, 189-197, 2009.

Karlický, M. and Hénoux, J. C.: Return current losses in pulse beam heating of the solar atmosphere, Astron. Astrophys., 264, 679685, 1992.

Karlický, M. and Hénoux, J. C.: Impact H-alpha line polarization and return current, Astron. Astrophys., 383, 713-718, 2002.

Karlický, M., Alexander, D., Brown, J. C., and MacKinnon, A. L.: Return current and collisional effects in nonthermal electron beams with pulsed injection, Sol. Phys., 129, 325-341, 1990.

Karlický, M., Kašparová, J., and Heinzel, P.: Collisional excitation and ionization of hydrogen by return current in solar flares, Astron. Astrophys, 416, L13-L16, 2004.

Karlický, M., Nickeler, D. H., and Bárta, M.: Particle-in-cell simulations of return current in solar flares, Astron. Astrophys., 486, 325-329, 2008.

Lee, K. W., Büchner, J., and Elkina, N.: Current driven lowfrequency electrostatic waves in the solar corona: Linear theory and nonlinear saturation, Phys. Plasmas., 14, 112903-112908, 2007.

Lee, K.W., Büchner, J., and Elkina, N.: Collisionless transport of energetic electrons in the solar corona at current-free double layers, Astron. Astrophys., 478, 889-895, 2008.

Michailovskij, A. B.: Teorija plazmennych neustojchivostej, Atomizdat, Moskva, 1975 (in Russian).

Niemiec, J., Pohl, M., Stroman, T., and Nishikawa, K.: Production of magnetic turbulence by cosmic rays drifting upstream of supernova remnant shocks, Astrophys. J., 684, 1174-1189, 2008.

Nishikawa, K.-I., Hardee, P. E., Richardson, G., Preece, R., Sol, H., and Fishman, G. J.: Particle acceleration in relativistic jets due to Weibel instability, Astrophys. J., 595, 555-563, 2003.

Nishikawa, K.-I., Hardee, P. E., Hededal, C. B., and Fishman, G. 
J.: Acceleration mechanics in relativistic shocks by the Weibel instability, Astrophys. J., 642, 1267-1274, 2006.

Rowland, H. L. and Vlahos, L.: Return currents in solar flares Collisionless effects, Astron. Astrophys., 142, 219-224, 1985.

Štepán, J. A., Kašparová, J., Karlický, M., and Heinzel, P.: Hydrogen Balmer line formation in solar flares affected by return currents, Astron. Astrophys., 472, L55-L58, 2007.

van den Oord, G.H.: The electrodynamics of beam/return current systems in the solar corona, Astron. Astrophys., 234, 496-518, 1990.
Weibel, E. S.: Spontaneously growing transverse waves in a plasma due to an anisotropic velocity distribution, Phys. Rev. Lett., 2, 83-84, 1959.

Zharkova, V. V., Brown, J. C., and Syniavskii, D. V.: Electron beam dynamics and hard $\mathrm{X}$-ray bremsstrahlung polarization in a flaring loop with return current and converging magnetic field, Astron. Astrophys., 304, 284-295, 1995. 